Check for updates

Cite this: RSC Adv., 2018, 8, 20630

Received 20th February 2018

Accepted 14th May 2018

DOI: $10.1039 / c 8 r a 01544 a$

rsc.li/rsc-advances

\section{Molecular dynamics and the translational- rotational coupling of an ionically conducting glass-former: amlodipine besylate $\uparrow$}

\author{
Safna Hussan K. P, D *a Mohamed Shahin Thayyil, ${ }^{\text {a }}$ S. K. Deshpande, ${ }^{\text {b }}$ Jinitha T. V, ${ }^{c}$ \\ Manoj $K^{d}$ and K. L. Ngai ${ }^{\mathrm{e}}$
}

We studied the conductivity relaxation originating from a glass-former composed of cations and anions, and the relation to the structural $\alpha$-relaxation at temperatures above and below the glass transition temperature. The material chosen was amorphous amlodipine besylate (AMB), which is also a pharmaceutical with a complex chemical structure. Measurements were made using differential scanning calorimetry (DSC), broadband dielectric spectroscopy (BDS) and X-ray diffraction, and the characterization was assisted using density functional theory (DFT). The X-ray diffraction pattern confirms the amorphous nature of vitrified AMB. Both the ionic and dipolar aspects of the dynamics of $A M B$ were examined using these measurements and were used to probe the nature of the secondary conductivity and dipolar relaxations and their relation to the conductivity $\alpha$-relaxation and the structural $\alpha$-relaxation. The coupling model predictions and quantum mechanical simulations were used side by side to reveal the properties and nature of the secondary conductivity relaxation and the secondary dipolar relaxation. Remarkably, the two secondary relaxations have the same relaxation times, and are one and the same process performing dual roles in conductivity and dipolar relaxations. This is caused by the translation-rotation coupling of the AMB molecule. Thus, AMB has both conductivity $\alpha$ - and $\beta$ relaxations, and application of the coupling model shows that these two relaxations are related in the same way as the structural $\alpha$-relaxation and the Johari-Goldstein $\beta$-relaxation are. This important result has an impact on the fundamental understanding of the dynamics of ionic conductivity.

\section{Introduction}

The dynamics of glass-forming liquids and glass transition is currently considered to be an important unsolved problem in condensed matter physics and physical chemistry. Experimental and theoretical efforts in the past few decades have made some advances but a solution to the problem is still needed. Part of the difficulty encountered in solving the problem is the existence of a variety of glass-formers with widely different chemical composition and physical structures, which give rise to diverse thermodynamic and dynamic properties to be reckoned with. However, these diverse properties are beneficial in practical applications in many areas including ionic

${ }^{a}$ Department of Physics, University of Calicut, Malappuram-673635, Kerala, India. E-mail: safna_dop@uoc.ac.in; safnahussain2@gmail.com

${ }^{b} U G C-D A E$ Consortium for Scientific Research, Mumbai Centre, BARC, Mumbai, 40085, India

${ }^{c}$ Department of Chemistry, University of Calicut, Malappuram-673635, Kerala, India ${ }^{d}$ College of Pharmaceutical Sciences, Govt. Medical College, Kozhikode-673008, Kerala, India

${ }^{e}$ CNR-IPCF, Largo Bruno Pontecorvo 3, I-56127, Pisa, Italy

$\dagger$ Electronic supplementary information (ESI) available. See DOI: $10.1039 / \mathrm{c} 8 \mathrm{ra} 01544 \mathrm{a}$ liquids, amorphous pharmaceuticals, biomolecules, biopolymers, metallic glasses, colloids etc. The synergy between basic research and research into the applications of glass-formers propels further studies of novel materials, and the research reported in this paper is such an example. The glass-former studied is an ionically conducting material as well as a pharmaceutical.

The molecular dynamics of glass-forming materials composed of cations and anions are interesting because of the presence of not only the structural relaxation responsible for glass transition and viscous flow, but also the ionic conductivity relaxation. ${ }^{1-6}$ The properties of the two kinds of relaxation are of current interest to the two corresponding research communities. If found, any relation between the two relaxations would considerably enrich the fundamental understanding of the dynamics. With this in mind, we studied an ionically conducting glass-former, amlodipine besylate (AMB), which is also a pharmaceutical for the treatment of hypertension in a similar way to nifedipine. ${ }^{7,8}$

In pharmaceutical applications ${ }^{9}$ of amorphous AMB, an important concern is crystallization. We have already reported the recrystallization tendencies of amorphous AMB in neat form during one month of storage at room temperature. 
Improved physical stability was achieved via the preparation of a binary formulation of AMB with the biocompatible excipient PVP K30. ${ }^{10}$ Notwithstanding, the present study of the molecular and ionic mobility in amorphous AMB will have a benefit in identifying the factors that govern devitrification in both the supercooled and glassy states. Thus our present study also is of interest to the pharmaceutical research community.

Even though the molecular relaxations responsible for crystallization vary from case to case, the molecular motion responsible for $\beta$-relaxation ${ }^{\mathbf{1 1 - 1 3}}$ is one of the governing factors for recrystallization in many amorphous pharmaceuticals. ${ }^{\mathbf{1 4}}$ Hence the study of the secondary dipolar and secondary ionic conductivity relaxations, and their connection to the primary relaxations, is relevant and important to several research communities.

The aim of the present work is to gain a better insight into the molecular and ionic dynamics of AMB with the use of various experimental methods. These include differential scanning calorimetry (DSC) and broadband dielectric spectroscopy (BDS) in the glassy and the supercooled liquid states. The measurements enable us to assess the glass-forming abilities and dynamic properties (such as fragility and nonexponentiality) of the primary $\alpha$-relaxation and the secondary dipolar and conductivity relaxations, as well as the relation between the structural relaxation and ionic conductivity relaxation in their properties. Computational calculations were also carried out to gain a better understanding of the relaxation processes in AMB. The results are of interest to the conductivity relaxation and glass transition communities because novel properties are encountered in this study.

\section{Experimental section}

\section{Materials}

Amlodipine besylate, a white crystalline powder with a diamond shape and a molecular weight of $567.1 \mathrm{~g} \mathrm{~mol}^{-1}$, was purchased from Alfa Aesar with $99.8 \%$ purity. It is a calcium antagonist and is chemically known as 3-ethyl-5-methyl-(4RS)-2-[(2-aminoethoxy) methyl]-4-(2-chlorophenyl)-6-methyl-1,4-dihydropyridine-3,5dicarboxylate benzene sulphonate. It was used without further purification.

\section{Methods}

Density functional theory. The geometrical calculations of AMB $\left(\mathrm{C}_{20} \mathrm{H}_{25} \mathrm{CIN}_{2} \mathrm{O}_{5} \cdot \mathrm{C}_{6} \mathrm{H}_{6} \mathrm{O}_{3} \mathrm{~S}\right)$ were performed using density functional theory using the Gaussian package with GaussView 05 as the visualization package. The initial geometry chosen for the calculation was taken from the PubChem database and optimised with the B3LYP/6-311++G (d,p) level of theory. In order to simulate the side chain rotation in $\mathrm{AMB}$, relaxed geometry scans of different available dihedral side chains were performed at the same level of theory. All the computational calculations were done in the gas phase, since no solvents were used during our experimental investigation. Transition states (TS) and minima (MIN) were further confirmed by performing vibrational analysis. ${ }^{15-17}$
X-ray diffraction (XRD). X-ray diffraction experiments were done in the range of $2 \theta$ from $20^{\circ}$ to $80^{\circ}$ at a speed rate of $10^{\circ}$ per minute at ambient temperature on a Rigaku Miniflex 600 powder diffractometer fitted with a curved crystal monochromator and attenuated total reflectance, which was equipped with Ni-filtered CuK $\alpha$ radiation $(\lambda=1.5418 \AA) .{ }^{18,19}$

Differential scanning calorimetry (DSC). The thermal properties of neat and binary AMB were examined using a Perkin Elmer DSC 4000 system. The DSC was calibrated for temperature and enthalpy using an indium standard. The samples were measured using aluminum pans. All measurements were carried out in the range of $303 \mathrm{~K}$ to $550 \mathrm{~K}$ with a heating rate of 5 $\mathrm{K} \min ^{-1}$. The crystallization, melting and glass transition temperatures were determined.

Broadband dielectric spectroscopy (BDS). The dielectric measurements of AMB were carried out using a Novo-Control Alpha dielectric spectrometer, in a wide frequency range $\left(10^{-2}\right.$ to $10^{7} \mathrm{~Hz}$ ) at temperatures from $203 \mathrm{~K}$ to $473 \mathrm{~K}$. The AMB was placed between two stainless steel electrodes (diameter $30 \mathrm{~mm}$ ), and Teflon spacers (thickness $0.1 \mathrm{~mm}$ ) were used to keep the sample confined between the electrodes even at high temperatures. Dielectric measurements of neat and binary AMB were taken on heating after fast vitrification. The temperature was controlled using a Quattro temperature controller with temperature stability better than $0.1 \mathrm{~K}$.

\section{Results and discussion}

\section{Geometry}

The distinctly complex structure of AMB was optimised using density functional theory with the B3LYP/6311-G++ level of theory. The quantum computational calculations predicted the stable structure of AMB and revealed its polar nature due to the presence of electropositive amlodipine cations with electronegative besylate anions. The optimised geometry, along with the electron densities in the molecular electrostatic potential

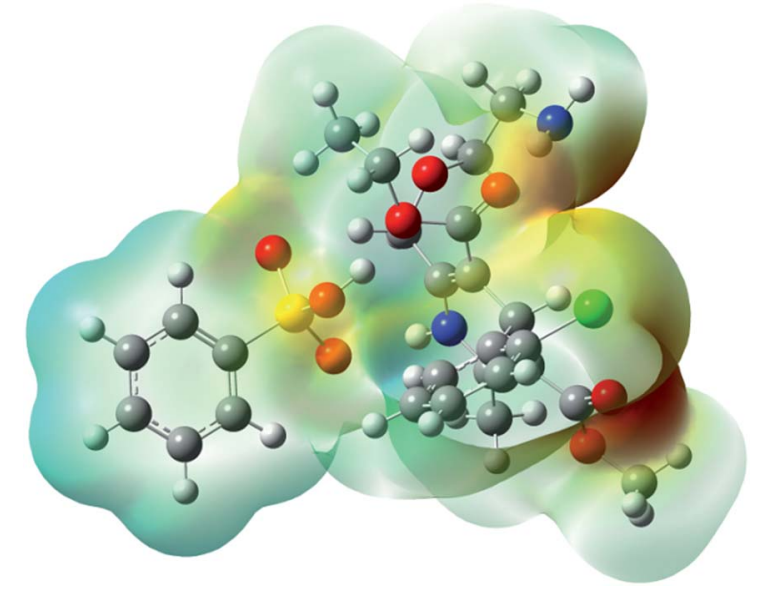

Fig. 1 The chemical three-dimensional structure of amlodipine besylate. Electron densities are presented to allow for comparison of the sizes of the ions. The red colour indicates the electronegative area while the blue colour indicates the electropositive region of AMB. 
map, is shown in Fig. 1 (ref. 18) as has been reported for other ionic liquids. ${ }^{17,20,21}$

\section{XRD pattern}

As can be seen from the X-ray diffraction patterns in Fig. 2, the crystalline structure of pure AMB reported previously in the patent of Ettema ${ }^{22}$ et al. is confirmed via the prominent characteristic peaks at diffraction angles of $5.8,11.6$, and $13.0^{\circ}(2 \theta)$ corresponding to the (002), (004), and (023) planes respectively. Meanwhile, the patterns of the AMB forms prepared via quench cooling exhibited a characteristic "amorphous halo" devoid of any sharp diffraction peaks.

\section{Thermal properties}

The DSC thermogram of crystalline AMB upon heating (10 $\mathrm{K} \mathrm{min}^{-1}$ ), as depicted in Fig. 3, shows an endothermic peak at $477 \mathrm{~K}$ corresponding to the melting of the sample, whereas amorphous AMB prepared via quench cooling presents its glass transition at $318 \mathrm{~K}^{\mathbf{1 , 2 1 , 2 3 - 2 5}}$

\section{Molecular dynamics}

The molecular mobility of AMB in the glassy and supercooled states was studied using BDS to provide detailed experimental evidence of the dynamics of glass transition beyond DSC. The isothermal dielectric measurements of AMB at ambient pressure were obtained from the temperature of the deep glassy state $(203 \mathrm{~K})$ to temperatures near the melting point $(473 \mathrm{~K})$ in a wide frequency window from $10^{-1}$ to $10^{7} \mathrm{~Hz}$. The dielectric loss of AMB over wide temperatures from the deep glassy to supercooled liquid states is shown in Fig. S1. $\dagger$ It was found that the spectra for all temperatures were dominated by ion conductivity relaxation due to the hopping of ions, especially the amlodipine cation and the besylate anion in AMB, making it impossible to observe the structural relaxation in the dielectric susceptibility. Even though the dielectric measurements can be represented by different frequency dependent complex quantities like the permittivity, $\varepsilon^{*}(f)=\varepsilon^{\prime}(f)-i \varepsilon^{\prime \prime}(f)$, the conductivity,

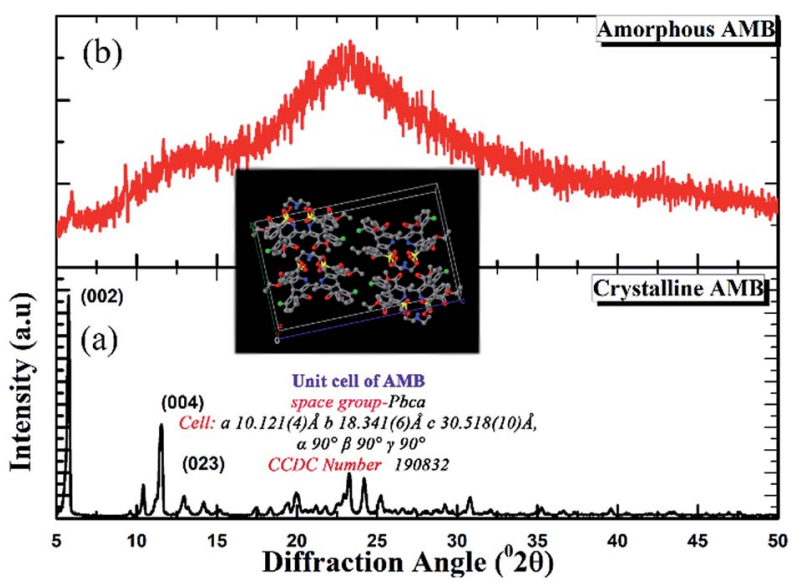

Fig. 2 XRD patterns of (a) pure crystalline $A M B$ and (b) vitrified $A M B$ after quench cooling.

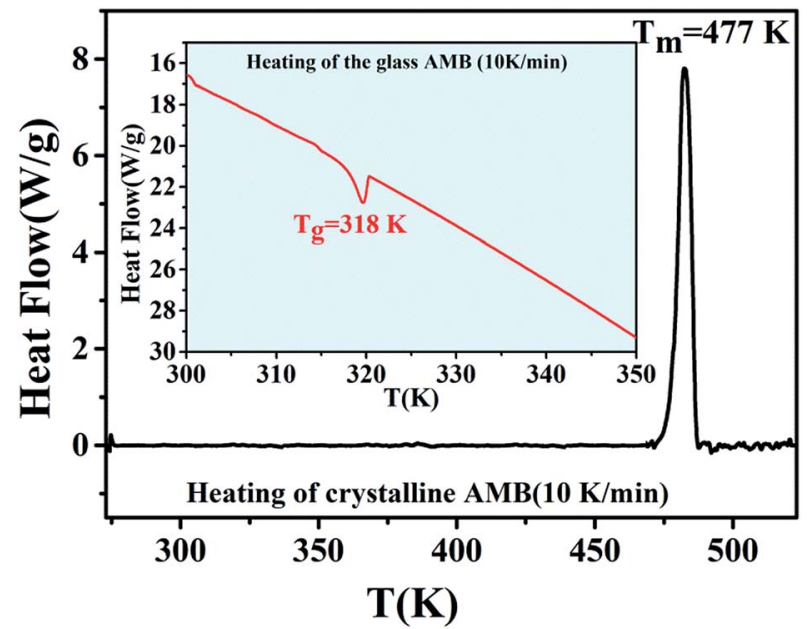

Fig. 3 DSC thermogram of AMB obtained during the melting of the crystalline form (heating rate $10 \mathrm{~K} \mathrm{~min}^{-1}$ ). The inset shows the glass transition temperature of amorphous AMB.

$\sigma^{*}(f)=\sigma^{\prime}(f)+\mathrm{i} \sigma^{\prime \prime}(f)$, and the electric modulus, $M^{*}(f) \equiv 1 / \varepsilon^{*}(f)=$ $M^{\prime}(f)+\mathrm{i} M^{\prime \prime}(f)$, all of them are equivalent. ${ }^{26,27}$ Following standard practice and in a similar way to what has been done before for ionic liquids, we considered data in the electric modulus representation, as shown in Fig. $4 .^{\mathbf{1 , 2 6 , 2 8 - 3 8}}$

The temperature dependence of the $\alpha$-conductivity relaxation time, the dispersion parameters, and the dc conductivity term at various temperatures were obtained from the fit of the experimental $M^{\prime \prime}(f)$ data using the Havriliak-Negami function. ${ }^{6}$

$$
\begin{aligned}
M^{*}(\omega) & =M^{\prime}(\omega)+\mathrm{i} M^{\prime \prime}(\omega)=\frac{1}{\varepsilon^{*}(\omega)} \\
& =M_{\alpha}+\frac{\Delta M}{\left(1+\left(-i\left(\omega \tau_{\sigma \alpha}\right)^{-1}\right)^{\alpha}\right)^{\beta}}
\end{aligned}
$$

Super-cooled liquid state. The electric loss modulus spectra $M^{\prime \prime}(f)$ of AMB above $T_{\mathrm{g}}$ are shown in Fig. 4. At first glance, only the $\alpha$-conductivity relaxation is observed in the super-cooled

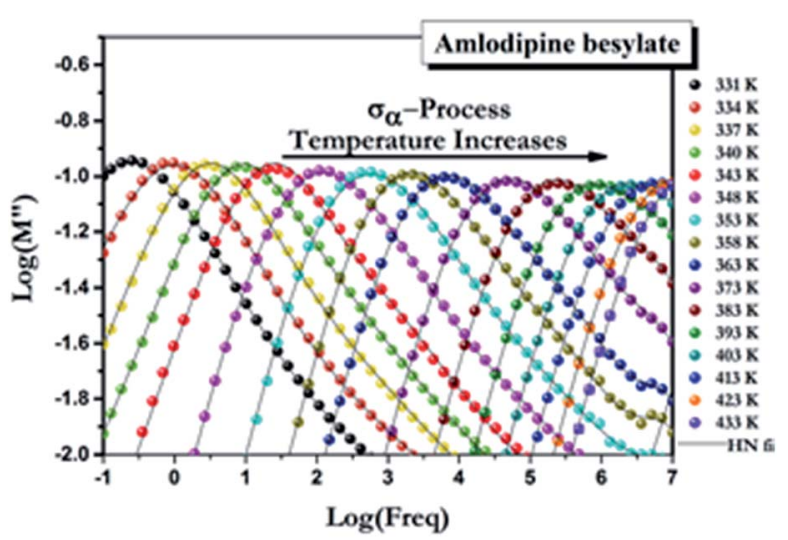

Fig. 4 Electric modulus spectra for AMB at ambient pressure collected at temperatures above the glass transition temperature. 
liquid state. As the temperature increases, the $\alpha$-conductivity loss peak moves to the higher frequency side.

In order to study the dependence of the $\alpha$-conductivity loss peak shape on temperature, the data taken at various temperatures from $337 \mathrm{~K}$ to $473 \mathrm{~K}$ were shifted to overlap with $343 \mathrm{~K}$ after normalizing the peak heights to unity, ${ }^{\mathbf{1 , 2 6 , 2 9 - 3 6}}$ and are shown in Fig. S2. $\dagger$ The trend of the decrease of the full-width at half-maximum (FWHM) of the $M^{\prime \prime}(f)$ loss peak with an increase in temperature is evident.

To characterize the nonexponential character of the conductivity relaxation, the $M^{\prime \prime}(f)$ loss peaks were fitted using the one-sided Fourier transform of the Kohlrausch-WilliamsWatts (KWW) ${ }^{1,25,26,29-36}$ function,

$$
\varphi(t)=\exp \left[-\left(\frac{t}{\tau_{\sigma \alpha}}\right)^{\beta_{\mathrm{KWw}}}\right],
$$

where $t$ is time, $\tau_{\sigma \alpha}$ is the characteristic conductivity $\alpha$-relaxation time and $\beta_{\mathrm{KWW}}$ is the stretching parameter. ${ }^{25,29,35,39}$ The fit of the data at 334 and $337 \mathrm{~K}$ is presented in Fig. S3† with $\beta_{\mathrm{KWW}}$ $\equiv\left(1-n_{\sigma}\right)=0.56$, indicating broad frequency dispersion, which may have some connection to the unstable nature of amorphous AMB.

Glassy state. Below the glass transition temperature, another loss in $M^{\prime \prime}(f)$ was clearly observed as shown in Fig. 5a, which may originate from the secondary conductivity relaxation as observed in procainamide $\mathrm{HCl},{ }^{26,31}$ lidocaine $\mathrm{HCl},{ }^{30}$ procaine $\mathrm{HCl}^{26}$ and chlorpromazine $\mathrm{HCl} .{ }^{15}$ Alternatively, it may be due to a secondary dipolar relaxation, and the only way to identify it is to represent the same data in terms of $\varepsilon^{\prime \prime}(f)$ as shown in Fig. $5 \mathrm{~b}$. As shown there, $\varepsilon^{\prime \prime}(f)$ also shows a loss peak, and thus the loss might originate from a secondary dipolar relaxation ${ }^{\mathbf{1 5}}$ with intermolecular or intramolecular character.

The dependence of the $\alpha$-conductivity relaxation time on temperature for neat AMB follows the time honoured VogelFulcher-Tammann (VFT) expression,

$$
\tau_{\sigma \alpha-\mathrm{M}}=\tau_{\infty} \exp \left[\frac{B}{T-T_{0}}\right]
$$

where $\tau_{\infty}, B$ and $T_{0}$ are fitting parameters. This property, like that found for other pharmaceutical glass-forming samples, ${ }^{7,39-43}$ is depicted in Fig. 6.

The temperature dependence of the relaxation time of the $\beta$ process can be described using the Arrhenius equation

$$
\tau_{\sigma \beta}=\tau_{\infty} \exp \left[\frac{E_{\mathrm{a}}}{R T}\right]
$$

and the activation energy $E_{\mathrm{a}}$ for the conductivity $\beta$-process is $38.9 \mathrm{~kJ} \mathrm{~mol}^{-1}$.

Only one well resolved secondary relaxation is observed below the glass transition temperature, which is detected either as a dipolar rotation in dielectric susceptibility or as an ion conductivity relaxation in the electric modulus. Interestingly, the secondary dipolar and conductivity relaxation times calculated in both scenarios superimpose perfectly, as is clearly seen in Fig. 6. These properties suggest the presence of a strong translation-rotation coupling of the molecules, leading to
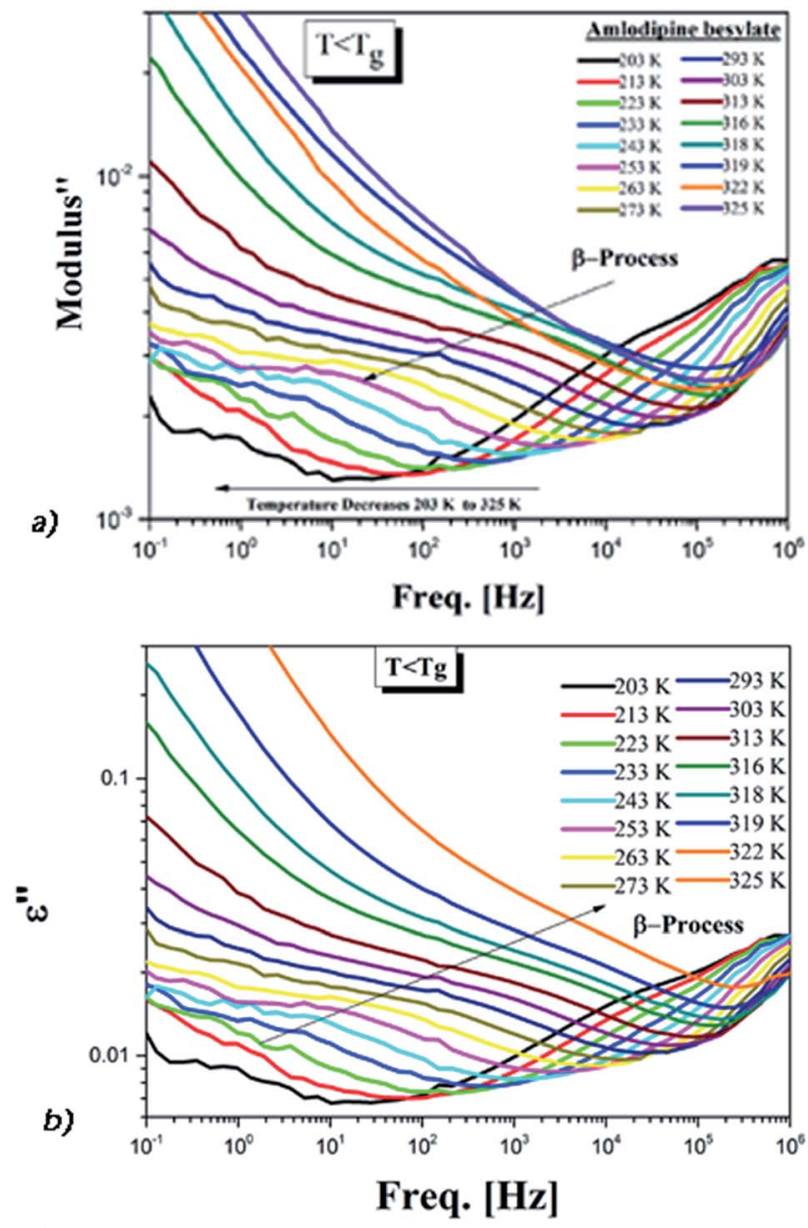

Fig. 5 (a) Secondary conductivity relaxation of AMB in the electric modulus. (b) The dielectric spectra of AMB below $T_{\mathrm{g}}$ for the same temperatures.

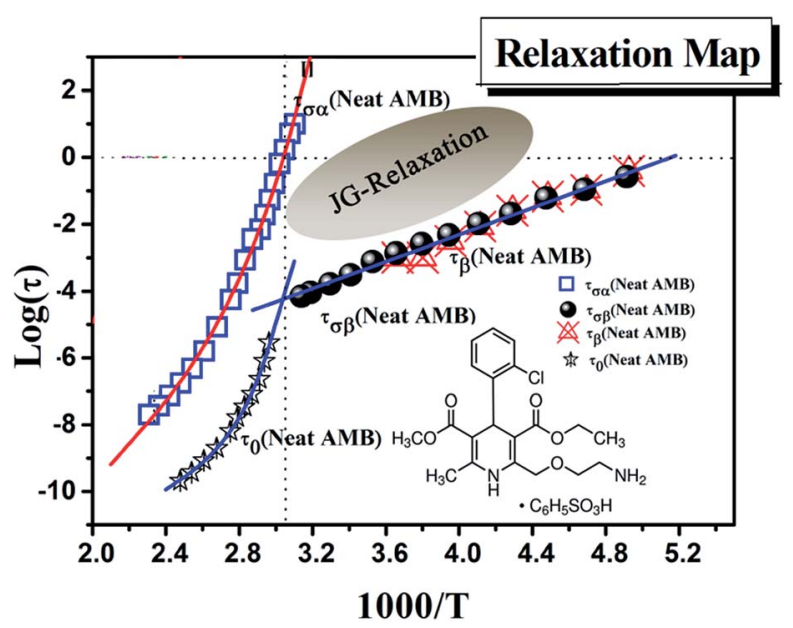

Fig. 6 Relaxation map obtained for neat AMB. All three processes including the $\alpha$-conductivity relaxation and the secondary relaxation are indicated together with the calculated primitive relaxation times obtained from the coupling model prediction. 
equivalent secondary conductivity and dipolar relaxation times. The latter can be considered as the Johari-Goldstein (JG) $\beta$ relaxation relating to the dipolar structural $\alpha$-relaxation, which is supposed to be a universal feature of glass-forming systems of all kinds. ${ }^{38,44}$ While the secondary conductivity relaxation is the precursor of the conductivity $\alpha$-relaxation, an analogue of the relation of the JG $\beta$-relaxation to the structural $\alpha$-relaxation has been found before in an ionic liquid. ${ }^{39}$ The fitting parameters of the VFT and the value of $T_{\mathrm{g}}$ obtained from BDS, as well as from DSC, have already been reported by Safna et al. ${ }^{10}$ Here, we focus more on the origin of the secondary relaxation found in AMB, and so the fitting parameters of the Arrhenius equations are tabulated in Table 1. In passing, it is worth pointing out that the translation-rotation coupling has been found before from the fact that the same $\alpha$-conductivity relaxation and $\alpha$-dipolar relaxation have the same relaxation time and frequency dispersion in two ionic liquids, which was studied using light scattering and dielectric spectroscopy by Pabst et al. ${ }^{45}$

However, the possibility of an intramolecular secondary relaxation due to the presence of a dihedral side chain with amine groups cannot be excluded. To resolve the issue and confirm the presence of the secondary conductivity relaxation, we used the Coupling Model (CM).$^{15,25,26,36,46}$ Originally, the CM was applied to secondary relaxations having properties strongly connected to the structural $\alpha$-relaxation. The existence and universal presence of this kind of secondary relaxation, called the Johari-Goldstein (JG) $\beta$-relaxation, was suggested by its analogy to the primitive relaxation of the CM, which started in a paper $^{47}$ in 1998, and has since been extensively developed. ${ }^{25,48-53}$ The $\mathrm{CM}^{54}$ has the following exact relation between the primitive relaxation time $\tau_{0}$ and the $\alpha$-relaxation time $\tau_{\alpha}$,

$$
\tau_{\alpha}=\left(t_{\mathrm{c}}{ }^{-n} \tau_{0}\right)^{1-n}
$$

where $t_{\mathrm{c}}$ is a constant $\approx 1$ ps for most molecular glass-formers, and $(1-n)$ is the exponent of the KWW correlation function of the $\alpha$-relaxation. Although similar, the JG $\beta$-relaxation and the primitive relaxation are not identical processes, and hence their relaxation times, $\tau_{\beta}(T)$ and $\tau_{0}(T)$, are not necessarily equal. Only approximate correspondence between their orders of magnitude can be expected or predicted, i.e., $\tau_{\beta}(T) \approx \tau_{0}(T)$. Thus $\tau_{0}(T)$ calculated from $\tau_{\alpha}(T)$ using the CM equation provides an order of magnitude estimate of $\tau_{\beta}(T)$ to compare with its experimental value. It is unfortunate that others ${ }^{55}$ have mistakenly taken $\tau_{\beta}(T)$ $\approx \tau_{0}(T)$ as an exact equality and made unnecessary and unjustified negative comments on one paper ${ }^{51,52}$ based on their own misconceptions.

The CM equation is generally applicable to relaxation and diffusion in all interacting systems, ${ }^{54}$ including not only

Table 1 Fitting parameters from the Arrhenius equation along with the barrier energy obtained from the molecular dynamic simulation

$\beta$-Process, Arrhenius

Sample $\quad \begin{array}{lll}\log \left(t_{\mathrm{a}}\right) & E_{\mathrm{a}}\left(\mathrm{kJ} \mathrm{mol}^{-1}\right) & E_{\mathrm{a}} \text { (barrier) }\left(\mathrm{kJ} \mathrm{mol}^{-1}\right)\end{array}$

$\begin{array}{llll}\text { AMB } & 10.41 \pm 0.2 & 38.9 \pm 0.05 & 33.0 \pm 5.0\end{array}$ structural relaxation but also ion conductivity relaxation because the molecules or ions are densely populated and interacting in both cases. For this reason, the CM also has long since been applied successfully to the ion conductivity relaxation of crystalline, glassy and molten ionic conductors..$^{15,25,26,36,46}$ There is also the CM equation relating the conductivity $\alpha$ relaxation time $\tau_{\sigma \alpha}(T)$ to the conductivity primitive relaxation time $\tau_{\sigma 0}(T)$, which is

$$
\tau_{\sigma \alpha}=\left(t_{\mathrm{c}}{ }^{-n_{\sigma}} \tau_{\sigma 0}\right)^{1-n_{\sigma}}
$$

where $t_{\mathrm{c}}$ is a constant $\approx 1$ ps for most ionic conductors, and ( $1-$ $n_{\sigma}$ ) is the exponent of the KWW function fitting the $M^{\prime \prime}$ data (presented in Fig. S3.†). The conductivity $\beta$-relaxation and the conductivity primitive relaxation are analogues but not identical, and hence their relaxation times, $\tau_{\sigma \beta}(T)$ and $\tau_{\sigma 0}(T)$, are approximately equal in their orders of magnitude, i.e., $\tau_{\sigma \beta}(T) \approx \tau_{\sigma 0}(T)$.

We have determined the $\tau_{\sigma \beta}(T)$ of AMB at temperatures below $T_{\mathrm{g}}$ from the electric modulus data in Fig. 5a and the plot is shown in Fig. 6, together with the line fitting the Arrhenius temperature dependence. Also shown in Fig. 6 are the values of $\tau_{\sigma 0}(T)$ calculated from the experimental values of $\tau_{\sigma \alpha}(T)$ above $T_{\mathrm{g}}$ using the CM equation, and the line is the fit to the temperature dependence of $\tau_{\sigma 0}(T)$ using the Vogel-Fulcher law. By extrapolating the Arrhenius dependence of $\tau_{\sigma \beta}(T)$ and the VogelFulcher dependence of $\tau_{\sigma 0}(T)$ both towards $T_{\mathrm{g}}$, as done in Fig. 6 , we verify the approximate agreement between $\tau_{\sigma 0}\left(T_{\mathrm{g}}\right)$ and $\tau_{\sigma \beta}\left(T_{\mathrm{g}}\right)$, and thus confirm that the conductivity $\beta$-relaxation present is in accord with the CM prediction as found before in other ionic conductors. ${ }^{15,25,26,36,46}$

From the good agreement of the secondary conductivity relaxation and the secondary dipolar relaxation times shown in Fig. 6, we have concluded that there is translation-rotation coupling. In principle, this can be confirmed by comparing the values of $\tau_{\sigma \alpha}(T)$ with the structural relaxation times $\tau_{\alpha}(T)$. However, unfortunately this cannot be done because the dielectric loss peaks of AMB are masked by the dc-conductivity that arises from the hopping of ions, as shown in Fig. S1. $\dagger$ In order to achieve this we may have to perform experiments at elevated pressure.

This provides strong support for identifying this secondary relaxation as the conductivity $\beta$-relaxation of $\mathrm{AMB}$, acting as the precursor of the conductivity $\alpha$-relaxation, as reported before for other ionic conducting pharmaceuticals, viz., procainamide $\mathrm{HCl},{ }^{26,31}$ lidocaine $\mathrm{HCl},{ }^{30}$ procaine $\mathrm{HCl},{ }^{26}$ chlorpromazine $\mathrm{HCl}^{15}$ and an ionic liquid. ${ }^{39}$

In order not to exclude the occurrence of an intramolecular secondary relaxation, we have pursued computer simulation studies of an AMB molecule by incorporating all possible sidechain rotations about various dihedral angles. Among them (the detailed report is shown in the ESI $\dagger$ ), the rotation around the third oxygen atom, as shown in Fig. 7, has a barrier energy of $33 \mathrm{~kJ} \mathrm{~mol}^{-1}$, which is comparable to the experimental activation energy of $38.9 \pm 0.05 \mathrm{~kJ} \mathrm{~mol}^{-1}$ for the secondary relaxation.

The energy conformation is plotted for each dihedral angle in Fig. S4. $\uparrow$ The calculated value of the activation energy may be slightly lower than that of the observed experimental values 


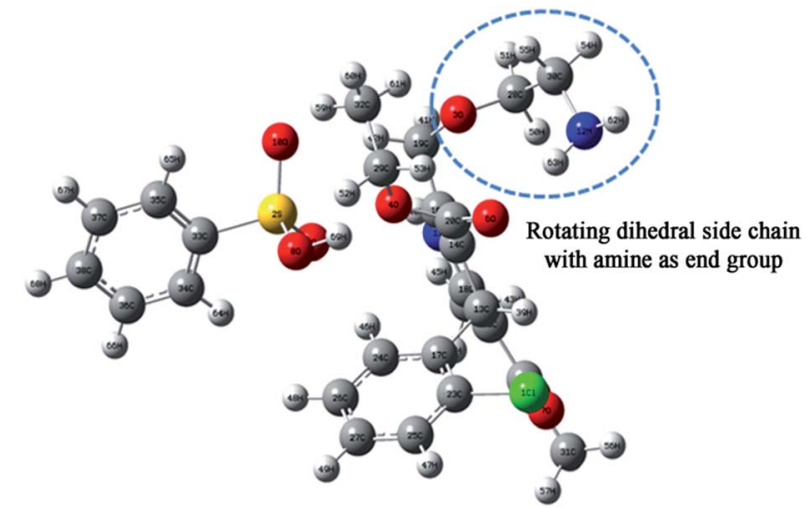

Fig. 7 Atomic arrangement of the amlodipine besylate $\mathrm{C}_{20} \mathrm{H}_{25} \mathrm{ClN}_{2}-$ $\mathrm{O}_{5} \cdot \mathrm{C}_{6} \mathrm{H}_{6} \mathrm{O}_{3} \mathrm{~S}$ structure. The rotating group is marked.

since we considered only a single molecule for the simulation. Even so, the obtained results are quite comparable within a tolerance of a few $\mathrm{kJ} \mathrm{mol}^{-1} \mathbf{. 1 5}^{15}$ Thus it is possible that the sidechain rotation is the origin of the secondary dipolar relaxation observed in $\varepsilon^{\prime \prime}(f)$, as shown in Fig. 5b. Despite this possibility, the origin of the observed secondary dipolar relaxation cannot be the dipolar JG process because the latter involves the motion of the entire AMB molecule and not a side group. ${ }^{42}$

\section{Summary and conclusion}

In this paper, the molecular dynamics of the neat amorphous drug AMB were investigated by means of various experimental techniques such as DSC, BDS, and XRD. Not only is AMB a good glass-former, but it is also an ionic conductor because of the amlodipine cations and the besylate anions. The molecular and ionic dynamics present in AMB offer an opportunity to study both the conductivity relaxations and structural relaxations and their precursors in the liquid and glassy states.

Crystallization is an important concern in the application of amorphous pharmaceuticals. Our experimental studies and characterization of the glassy state revealed that amorphous AMB does not have any re-crystallization tendencies on heating and cooling, but during storage it showed re-crystallisation tendencies. This result is relevant for the use of AMB as an active pharmaceutical ingredient in terms of suppressing crystallization tendencies initiated from the secondary relaxation.

On the other hand, the study of the conductivity relaxation and the analysis of the data using electric modulus representation enables the observation and characterization of the conductivity $\alpha$-relaxation responsible for d.c. conductivity in the liquid state above $T_{\mathrm{g}}$, and a conductivity $\beta$-relaxation in the glassy state. This identification is supported by the approximate agreement between its relaxation time $\tau_{\sigma \beta}$ and the primitive relaxation time $\tau_{\sigma 0}$ of the CM calculated from eqn (6). By confirming the presence of the conductivity $\beta$-relaxation and having its relaxation time $\tau_{\sigma \beta}$ bear a non-trivial relation to $\tau_{\sigma \alpha}$ similar to $\tau_{\sigma 0}$, we have found further evidence of the evolution of the ion conductivity dynamics with time starting from the primitive relaxation or $\beta$-relaxation and terminating in the many-ion $\alpha$ relaxation..$^{39,41}$ Thus, the conductivity $\alpha$-relaxation originates from the $\beta$-relaxation, and that means no ionic conductivity theory is complete and fundamental without taking into consideration the relation between the two relaxations.

Incidentally, analysis of the same data in the dielectric susceptibility representation yields evidence of the presence of a secondary dipolar relaxation with a comparable relaxation time and activation energy in the glassy state. Translationrotation coupling of AMB possibly explains the dual conductivity and dipolar nature of the secondary relaxation.

Notwithstanding, from the molecular dynamics simulation of the side chain rotation of $\mathrm{AMB}$, we found that the activation energy of one of the dihedral side chains with an amine group as one end is in good agreement with the experimental value of the activation energy of the secondary dipolar relaxation. Although this can be coincidental, the possibility of the observed secondary dipolar relaxation being an intramolecular secondary relaxation of the cation cannot be excluded at this time. One possible way to resolve this problem is to take measurements at elevated pressure in the future to investigate the pressure dependence of the secondary dipolar relaxation. If its relaxation time is pressure sensitive, then the possibility of it being the side chain rotation can be eliminated. In that case, due to the translation-rotation coupling, the secondary dipolar relaxation must be the JohariGoldstein $\beta$-relaxation of the structural $\alpha$-relaxation.

\section{Conflicts of interest}

There are no conflicts to declare.

\section{Acknowledgements}

The authors thankfully acknowledge the fruitful discussions held with Raj Suryanarayanan (Department of Pharmaceutics, University of Minnesota), G. Govindraj (Dean, Pondichery University) and Vijisha K Rajan. KPSH acknowledges UGCMANF for the fellowship with sanction number MANF-201718-KER-78598. KPSH and MST gratefully acknowledge the collaborative research grant from UGC-DAE (Sanction Order NoUDCSR/MUM/AO/CRS-M-2I0/2015/501 dated 06/01/2015). MST further acknowledges the financial assistance from KSCSTE (SRS, SARD), UGC (MRP) and DST FIST.

\section{Notes and references}

1 Z. Wojnarowska, K. Kołodziejczyk, K. J. Paluch, L. Tajber, K. Grzybowska, K. L. Ngai and M. Paluch, Phys. Chem. Chem. Phys., 2013, 15, 9205-9211.

2 J. R. Sangoro, C. Iacob, A. Serghei, C. Friedrich and F. Kremer, Phys. Chem. Chem. Phys., 2009, 11, 913-916.

3 P. Migowski, D. Zanchet, G. Machado, M. A. Gelesky, S. R. Teixeira and J. Dupont, Phys. Chem. Chem. Phys, 2010, 12, 1648.

4 M. J. Marczewski, B. Stanje, I. Hanzu, M. Wilkening and P. Johansson, Phys. Chem. Chem. Phys., 2014, 16, 12341-12349.

5 K. Lunstroot, K. Driesen, P. Nockemann, L. Viau, P. H. Mutin, A. Vioux and K. Binnemans, Phys. Chem. Chem. Phys., 2010, 12, 1879-1885. 
6 M. Schmollngruber, C. Schröder and O. Steinhauser, Phys. Chem. Chem. Phys., 2014, 16, 10999-11009.

7 E. Kaminska, M. Tarnacka, K. Kaminski, K. L. Ngai and M. Paluch, Eur. J. Pharm. Biopharm., 2015, 97, 185-191.

8 T. El Goresy and R. Böhmer, J. Non-Cryst. Solids, 2006, 352, 4459-4463.

9 K. L. Grzybowska, K. Paluch, M. Grzybowski, A. Wojnarowska, Z. Hawelek, L. Kołodziejczyk and K. Ngai, J. Phys. Chem. B, 2010, 114(2010), 12792-12801.

10 K. P. Safna Hussan, M. S. Thayyil, S. K. Deshpande, T. V. Jinitha, K. Manoj and K. L. Ngai, Eur. J. Pharm. Sci., 2018, 119, 268-278.

11 S. Capaccioli, K. L. Ngai and N. Shinyashiki, J. Phys. Chem. B, 2007, 111, 8197-8209.

12 K. L. Ngai, J. Non-Cryst. Solids, 2006, 352, 404-408.

13 S. Capaccioli, K. Kessairi, M. S. Thayyil, D. Prevosto and M. Lucchesi, J. Non-Cryst. Solids, 2011, 357, 251-257.

14 K. Adrjanowicz, Z. Wojnarowska, P. Wlodarczyk, K. Kaminski, M. Paluch and J. Mazgalski, Eur. J. Pharm. Sci., 2009, 38, 395-404.

15 S. Hensel-bielowka, K. L. Ngai, A. Swiety-pospiech, L. Hawelek, J. Knapik, W. Sawicki and M. Paluch, J. NonCryst. Solids, 2015, 407, 81-87.

16 K. G. Sangeetha, K. K. Aravindakshan and K. P. Safna Hussan, J. Mol. Struct., 2017, 1150, 135-145.

17 K. P. Safna Hussan, T. M. Shahin, K. R. Vijisha and K. Muraleedharan, Comput. Biol. Chem., 2018, 72, 113-121.

18 K. Safna Hussan, T. Mohamed Shahin, S. K. Deshpande and T. V. Jinitha, AIP Conf. Proc., 2017, 1832, 070011.

19 K. P. Safna Hussan, T. Mohamed Shahin, S. K. Deshpande and T. V. Jinitha, AIP Conf. Proc., 2017, 1832, 070011.

20 K. G. Sangeetha, K. K. Aravindakshan and K. P. Safna Hussan, J. Mol. Struct., 2017, 1150, 135-145.

21 B. Katharina, R. Christiaan, N. Mark and R. Robin D, In search of pure liquid salt forms of aspirin: ionic liquid approaches with acetylsalicylic acid and salicylic acid, Phys. Chem. Chem. Phys., 2010, 12, 2011-2017.

22 G. J. B. Ettema, H. Hoorn and J. M. Lemmens, US6828339B2; K. Mereiter, J. Roll, Priv. Commun. to CCDC Depos. no. 190882, 2002, 2004, (23).

23 L. X. Scattering, S. N. Scattering, M. Matsugami, T. Takamuku, T. Otomo and T. Yamaguchi, J. Phys. Chem. B, 2006, 110, 12372-12379.

24 J. Sangster, J. Phys. Chem. Ref. Data, 1999, 28, 889-930.

25 K. L. Ngai, J. Phys.: Condens. Matter, 2000, 12, 6437-6451.

26 Z. Wojnarowska, K. Grzybowska, L. Hawelek and M. Paluch, J. Chem. Phys., 2012, 164507, 1-8.

27 F. Kremer and A. Schönhals, Broadband Dielectric Spectroscopy, 2003.

28 K. P. Safna Hussan, T. Mohamed Shahin, S. K. Deshpande, T. V. Jinitha, K. R. Vijisha and K. L. Ngai, J. Mol. Liq., 2016, 223, 1333-1339.

29 Z. Wojnarowska, M. Paluch, A. Grzybowski, K. Adrjanowicz, K. Grzybowska, K. Kaminski, P. Wlodarczyk and J. Pionteck, J. Chem. Phys., 2009, 131, 104505-104514.
30 Z. Wojnarowska, J. Pionteck, S. Pawlus, A. Grzybowski, Z. Wojnarowska, J. Pionteck, S. Pawlus and A. Grzybowski, J. Chem. Phys., 2012, 224501, 1.

31 Z. Wojnarowska, C. M. Roland, K. Grzybowska and M. Paluch, Phys. Rev. Lett., 2012, 015701, 1-4.

32 A. Swiety-Pospiech, Z. Wojnarowska, S. Hensel-Bielowka, J. Pionteck and M. Paluch, J. Chem. Phys., 2013, 138, 204502, 1-8.

33 T. Psurek, S. Hensel-Bielowka, J. Ziolo and M. Paluch, J. Chem. Phys., 2002, 116, 9882-9888.

34 J. Scheers, L. Niedzicki, G. Z. Zukowska, P. Johansson, W. Wieczorek and P. Jacobsson, Phys. Chem. Chem. Phys., 2011, 13, 11136-11147.

35 A. Rivera, A. Brodin, A. Pugachev and E. A. Rössle, J. Chem. Phys., 2007, 126, 0-7.

36 Z. Wojnarowska, K. L. Ngai and M. Paluch, J. Chem. Phys., 2014, 140, 1-6.

37 K. P. Safna Hussan, T. Mohamed Shahin, M. Binesh, S. K. Deshpande, K. R. Vijisha, S. H. Safna, M. S. Thayyil, M. Binesh, S. K. Deshpande and V. K. Rajan, J. Mol. Liq., 2018, 251, 487-491.

38 K. P. Safna Hussan, T. Mohamed Shahin, S. K. Deshpande, T. V. Jinitha and K. Jayant, Solid State Ionics, 2017, 310, 166-175.

39 K. Adrjanowicz, K. Kaminski, M. Paluch, P. Wlodarczyk, K. Grzybowska, Z. Wojnarowska, L. Hawelek, W. Sawicki, P. Lepek and R. Lunio, J. Pharm. Sci., 2010, 99, 828-839.

40 U. Sailaja, T. Mohamed Shahin, N. S. K. Kumar and G. Govindaraj, J. Pharm. Anal., 2014, 1-9.

41 T. H. Kim, T. Shibata, S. Kojima, D. M. Shin, Y. H. Hwang and J. H. Ko, Curr. Appl. Phys., 2014, 14, 965-969.

42 U. Sailaja, M. Shahin Thayyil, N. S. Krishna Kumar and G. Govindaraj, Eur. J. Pharm. Sci., 2013, 49, 333-340.

43 M. T. O. Abe, N. T. Correia, L. Valdes, J. M. B. Ndjaka and F. Affouard, J. Mol. Liq., 2015, 205, 74-77.

44 M. S. Thayyil, S. Capaccioli, D. Prevosto and K. L. Ngai, Philos. Mag., 2008, 88, 4007-4013.

45 F. Pabst, J. Gabriel, P. Weigl and T. Blochowicz, Chem. Phys., 2017, 494, 103-110.

46 G. Jarosz, M. Mierzwa, J. Ziozo, M. Paluch, H. Shirota and K. L. Ngai, J. Phys. Chem. B, 2011, 115, 12709-12716.

47 K. L. Ngai, J. Chem. Phys., 1998, 109, 6982-6994.

48 K. L. Ngai and M. Paluch, J. Chem. Phys., 2004, 120, 857-873. 49 S. Capaccioli, M. Paluch, D. Prevosto, L.-M. Wang and K. L. Ngai, J. Phys. Chem. Lett., 2012, 3, 735-743.

50 M. S. Thayyil, K. L. Ngai, D. Prevosto and S. Capaccioli, J. Non-Cryst. Solids, 2015, 407, 98-107.

51 K. L. Ngai, J. Habasaki, D. Prevosto, S. Capaccioli and M. Paluch, J. Chem. Phys., 2012, 137, 034511.

52 K. L. Ngai, J. Habasaki, D. Prevosto, S. Capaccioli and M. Paluch, J. Chem. Phys., 2014, 140, 019901.

53 K. L. Ngai and M. Paluch, J. Non-Cryst. Solids, 2017, 478, 1-11. 54 K. L. Ngai, Relaxation and Diffusion in Complex Systems, Springer, New York, 2011.

55 R. Casalini and C. M. Roland, Macromolecules, 2013, 46, 6364-6372. 